\title{
Astragaloside IV Attenuates Lipopolysaccharides-Induced Pulmonary Epithelial Cell Injury through Inhibiting Autophagy
}

\author{
Biwang Liu ${ }^{a}$ Huan Zhao $^{\mathrm{b}}$ Yonghui Wang ${ }^{\mathrm{a}}$ Huizhong Zhang ${ }^{\mathrm{c}}$ Yanmiao Mad \\ ${ }^{a}$ Experimental Management Center, Shanxi University of Chinese Medicine, Jinzhong, China; ${ }^{b}$ Department of \\ Pharmacy, The Hospital of Shanxi University of TCM, Taiyuan, China; ' Department of Scientific Research and \\ International Science and Technology Cooperation, Shanxi University of Chinese Medicine, Taiyuan, China; ${ }^{\mathrm{d}}$ School of \\ Basic Medical Sciences, Shanxi University of Chinese Medicine, Taiyuan, China
}

\section{Keywords}

Astragaloside IV · Acute respiratory distress syndrome ·

Autophagy · Lung injury · Pharmaceutical effect

\begin{abstract}
Background: Astragaloside IV has shown its promising effect on acute respiratory distress syndrome (ARDS). Objectives: We aim to explore whether astragaloside IV is effective for ARDS treatment in a lipopolysaccharides (LPS)-induced cell model and whether autophagy is involved in the therapeutic function of astragaloside IV. Methods: MLE-12 cells were induced by LPS to construct an ARDS model in vitro. Cell viability was estimated by cell counting kit- 8 and cell apoptosis by flow cytometry. Lactate dehydrogenase (LDH), malondialdehyde (MDA) and superoxide dismutase (SOD) levels were measured by enzyme-linked immunosorbent assay kit. The expression of tumour necrosis factor (TNF)-a, interleukin (IL)-6, zonula occludens (ZO)-1, Beclin-1 and autophagy-related (atg) 5 mRNA was evaluated by quantitative PCR, and the expression of ZO-1, microtubule-associated proteins 1A/1B light chain 3B (LC3B) I and, LC3B II protein by Western blot. Results: LPS effectively inhibited cell viability and LC3B I expression and enhanced LC3B II, Beclin-1 and atg5 expressions in MLE-12 cells. In LPS-induced ARDS cell model, astragaloside IV up-regulated cell viability, SOD ac-
\end{abstract}

tivity and ZO-1 and LC3B I expressions but down-regulated cell apoptosis, TNF-a, IL-6, LC3B II, Beclin-1 and atg5 expressions and LDH and MDA levels. 3-methyladenine promoted cell viability and ZO-1 expression, down-regulated Beclin-1 and atg5 expression, while Rapamycin (Rap) had an opposite effect. Astragaloside IV suppressed cell viability and ZO-1 expression after the Rap treatment. Conclusions: Astragaloside IV might suppress autophagy initiation directly or indirectly through suppressing the oxidative stress and inflammatory response, which further enhances the cell viability and tight junction and reduces apoptosis in LPS-stimulated pulmonary endothelial ARDS cell model, thus exerting its therapeutic function in ARDS.

๑ 2019 S. Karger AG, Basel

\section{Introduction}

Acute respiratory distress syndrome (ARDS), which is an acute pulmonary inflammatory syndrome, is characterized by the diffuse inflammation in the lung parenchyma and osmotic pulmonary oedema [1]. Gram-negative bacterial infection with lipopolysaccharides (LPS) is the main cause of ARDS [1]. LPS inducing the production of pro-inflammatory mediators such as TNF- $\alpha$ and interleukin (IL)-6 was believed to be involved in the pathogen-

\section{KARGER}

(c) 2019 S. Karger AG, Basel 
esis of ARDS and is widely used as experimental approaches in vitro and in vivo to explore the pathogenesis of ARDS [2-4]. LPS can activate inflammatory signaling pathways such as the nuclear factor-kappa $B$ pathway and induce the production of various inflammatory mediators such as nitric oxide, prostaglandins and pro-inflammatory cytokines, thereby mediating inflammatory injury [5]. LPS induces the production of cellular reactive oxygen species (ROS) and increases oxidative stress and is involved in the mechanism of ARDS [6]. LPS induces the expression of the key factors that endothelial tight junctions, and enhances extracellular permeability and inflammation, which are believed to be involved in the pathogenesis of ARDS [7]. In addition, studies have shown that LPS can induce autophagy in alveolar macrophages through Toll-like receptor 4, promote ROS production, and participate in the occurrence of ARDS [8]. Although it have been confirmed that inflammatory response, tight junction and oxidative stress play important roles in the pathogenesis of ARDS, however, apart from mechanical ventilation serving as a supportive treatment method, the complexity of ARDS mechanism results in a lack of effective clinical drug in improving the mortality of the disease [9-11].

Autophagy is a highly conserved physiological process that maintains homeostasis and adapts to the alteration of the microenvironment in eukaryotic cells [12]. In the case of starvation, hypoxia or toxic molecular stimulation, the cell initiates an autophagy process that mediated by lysosome-mediated digestion and intracellular component recycling to cope with adverse environments [12]. Recently, researchers showed that autophagy also plays an important role in ARDS caused by infection [13]. Rojo de la Vega et al. [14] demonstrated that autophagy could serve as a preventive and therapeutic intervention for ARDS.

Astragaloside IV is a monomer extracted from astragalus [15]. Modern pharmacology proved that astragalus has a variety of medicinal properties such as anti-inflammatory, immune-regulatory, anti-oxidative, anti-cancer, anti-diabetes, myocardial-protective, liver-protective and anti-viral functions [15]. Zhang et al. [16] found that pretreating the Parkinson's cell model with astragaloside IV significantly reversed the viability, nuclear pyknosis, ROS production and the apoptosis of injured cells. Recently, Chen et al. [17] demonstrated that astragaloside IV could protect paraquat-induced lung injury in mice. Thus, in this study, we explore the potential application of astragaloside IV in controlling the development of ARDS.

In the current study, we aim to explore whether astragaloside IV is effective in treating ARDS in the LPS- induced ARDS cell model and explore whether autophagy is involved in the therapeutic function of astragaloside IV. Our research provides a reference to promoting the application of astragaloside IV in ARDS.

\section{Materials and Methods}

\section{Cell Culture}

MLE-12, which is a transformed clonal line representative of alveolar lung epithelial type II cells [18], was purchased from American Type Culture Collection (USA). The cells were cultured in hydrocortisone, insulin, transferrin, oestrogen medium containing RPMI1640 (Thermo Fisher, Waltham, MA, USA), 2\% foetal bovine serum (FBS, Thermo Fisher, Waltham, MA, USA), $5 \mathrm{mg} / \mathrm{mL}$ insulin, $10 \mathrm{mg} / \mathrm{mL}$ transferrin, $30 \mathrm{nmol} / \mathrm{L}$ sodium selenite, $10 \mathrm{nmol} / \mathrm{L}$ hydrocortisone, $10 \mathrm{nmol} / \mathrm{L}$ b-estradiol (Sigma-Aldrich, St. Louis, MO, USA) with $50 \mathrm{U} / \mathrm{mL}$ penicillin and $50 \mu \mathrm{g} / \mathrm{mL}$ streptomycin (Thermo Fisher, Waltham, MA, USA). The cells were cultured in $5 \% \mathrm{CO}_{2}$ in a humid incubator at $37^{\circ} \mathrm{C}$ and passaged 3 times per week.

\section{Experiment Design}

Effect of LPS on Cell Viability and Autophagy in Pulmonary Epithelium

To observe the effect of LPS on cell viability and autophagy in MLE-12 cells, the cells were divided into 6 groups, respectively, treated with $0,10,25,50,100,200 \mathrm{ng} / \mathrm{mL}$ LPS (L2630, Sigma-Aldrich, St. Louis, MO, USA). The cell viability, microtubule-associated proteins $1 \mathrm{~A} / 1 \mathrm{~B}$ light chain $3 \mathrm{~B}$ (LC3B) I, LC3B II, Beclin-1 and autophagy-related (atg) 5 expressions were detected.

\section{Effect of Astragaloside IV on Cell Viability in Pulmonary} Epithelium

To observe the effect of astragaloside IV (Y0001171, $\mathrm{C}_{41} \mathrm{H}_{68} \mathrm{O}_{14}$, molecule weight: 784.97, CAS number: 84687-43-4, Sigma-Aldrich, St. Louis, MO, USA) on cell viability, the cells were also divided into 6 groups respectively treated by $0,5,10,20,40,80 \mu \mathrm{g} / \mathrm{L}$ astragaloside IV.

\section{Effect of Astragaloside IV on LPS-Stimulated Pulmonary}

Epithelium

To observe the effect of astragaloside IV on LPS-stimulated pulmonary epithelium, the cells were divided into 5 groups, namely, control (without treatment), LPS (cell treated by $100 \mathrm{ng} / \mathrm{mL}$ LPS as previously described [19]), LPS + 10 (cell treated by $100 \mathrm{ng} / \mathrm{mL}$ LPS and $10 \mu \mathrm{g} / \mathrm{L}$ astragaloside IV), LPS + 20 (cells treated by $100 \mathrm{ng} / \mathrm{mL}$ LPS and $20 \mu \mathrm{g} / \mathrm{L}$ astragaloside IV) and LPS +40 (cells treated by $100 \mathrm{ng} / \mathrm{mL}$ LPS and $40 \mu \mathrm{g} / \mathrm{L}$ astragaloside IV).

Role of Autophagy in the Effect of LPS

To investigate the role of autophagy in the effect of LPS, the cells were divided into control, LPS, LPS + 3-methyladenine (MA; M9281, Sigma-Aldrich, St. Louis, MO, USA), 3-MA, LPS + rapamycin (Rap; V900930, Sigma-Aldrich, St. Louis, MO, USA) and Rap groups, which were respectively treated by $100 \mathrm{ng} / \mathrm{mL}$ LPS, $100 \mathrm{ng} / \mathrm{mL}$ LPS with $3 \mathrm{mmol} / \mathrm{L} 3-\mathrm{MA}$ (autophagy inhibitor, pretreated $2 \mathrm{~h}$ before LPS induction), $3 \mathrm{mmol} / \mathrm{L} 3-\mathrm{MA}, 100 \mathrm{ng} / \mathrm{mL}$ LPS and $100 \mathrm{nmol} / \mathrm{L}$ Rap (autophagy inducer, pretreated $2 \mathrm{~h}$ before LPS induction) and $100 \mathrm{nmol} / \mathrm{L}$ Rap. 
Role of Autophagy in the Effect of Astragaloside IV on LPSStimulated Pulmonary Epithelium

To investigate the role of autophagy in the effect of astragaloside IV on LPS-stimulated pulmonary epithelium, the cells were divided into control, LPS, LPS + 20, LPS + $20+3$-MA and LPS + $20+$ Rap groups, which were respectively treated by $100 \mathrm{ng} / \mathrm{mL}$ LPS, $100 \mathrm{ng} / \mathrm{mL}$ LPS with $20 \mu \mathrm{g} / \mathrm{L}$ astragaloside IV , $100 \mathrm{ng} / \mathrm{mL} \mathrm{LPS}$ with $20 \mu \mathrm{g} / \mathrm{L}$ astragaloside IV and $3 \mathrm{mmol} / \mathrm{L} 3-\mathrm{MA}$, and $100 \mathrm{ng} /$ $\mathrm{mL}$ LPS with $20 \mu \mathrm{g} / \mathrm{L}$ astragaloside IV and $100 \mathrm{nmol} / \mathrm{L}$ Rap.

\section{Cell Counting Kit-8}

Cell viability was determined by cell counting kit-8 (CCK-8). In this study, $1 \times 10^{6} /$ well cells were seeded in a 96-well plate and treated as aforementioned for $24 \mathrm{~h}$ at $37^{\circ} \mathrm{C}$. After that, CCK-8 (HY-K0301, MedChemExpress, Monmouth Junction, NJ, USA) was used to determine the cell viability. Cell suspension was mixed with CCK- 8 solution (supplied by kit) and the optical density was read using a microplate reader (Multiskan, Thermo, Waltham, $\mathrm{MA}, \mathrm{USA}$ ) at $450 \mathrm{~nm}$. All procedures were conducted according to the protocols.

\section{Apoptosis Assessment}

Cell apoptosis rate was determined by ApoDETECT ${ }^{\mathrm{TM}}$ ANNEXIN V-FITC KIT (33-1,200, Thermo Fisher, Waltham, MA, USA). Cells were spun down at 3,000 rpm for $1 \mathrm{~min}$ and the supernatant was discarded. The cells were then washed with ice cold PBS, and the remaining PBS was removed using pipettor. The cells were resuspended in $1 \times$ binding buffer $(10 \mathrm{mmol} / \mathrm{L}$ Hepes/ $\mathrm{NaOH}, \mathrm{pH} 7.4,140 \mathrm{mmol} / \mathrm{L} \mathrm{NaCl}, 2.5 \mathrm{mmol} / \mathrm{L} \mathrm{CaCl}$, filtered by $0.2 \mu \mathrm{m}$ porefilter) to the density of $2.5 \times 10^{5}$ cells $/ \mathrm{mL}$. One hundred ninety micro litre cell suspension was added into $10 \mu \mathrm{L}$ Annexin V-FITC and incubated for $10 \mathrm{~min}$ at room temperature. The cells were washed by $1 \times$ binding buffer and then spun down for $1 \mathrm{~min}$ at 3,000 rpm and then resuspended in $190 \mu \mathrm{L}$ binding buffer after discarding the supernatant. The cells were added with $10 \mu \mathrm{L} 20 \mu \mathrm{g} / \mathrm{mL}$ propidium iodide stock solution and analyzed by flow cytometry (BD FACScanto II, Becton Dickinson, San Jose, CA, USA).

\section{Enzyme-Linked Immunosorbent Assay}

The whole cell proteins were collected and extracted using icecold RIPA buffer containing protease inhibitors (ab65621, Abcam, San Francisco, CA, USA). Lactate dehydrogenase (LDH) Assay kit (ab102526, Abcam, San Francisco, CA, USA), Lipid Peroxidation malondialdehyde (MDA) Assay kit (ab118970, Abcam, San Francisco, CA, USA), superoxide dismutase (SOD) Activity Assay kit (ab65354, Abcam, San Francisco, CA, USA) were then used to respectively determine the levels of $\mathrm{LDH}, \mathrm{MDA}$ and SOD according to the protocols.

\section{Quantitative PCR}

Total RNA was extracted using Trizol (Invitrogen, Waltham, MA, USA) and quantified by NanoDrop (Thermo Fisher Scientific, Waltham, MA, USA). RNA was reverse-transcribed into cDNA using iScriptTMcDNA Synthesis Kit (Bio-Rad, Hercules, CA, USA). Each sample was subjected to quantitative PCR (qPCR) program with a Fast Start Universal SYBR Green Master kit (Roche, Basel, Switzerland). qPCR was performed in ABI StepOne system (Applied BioSystems, Foster City, CA, USA), and $2.5 \mu \mathrm{L}$ cDNA template, $1 \mu \mathrm{L}$ forward primer, $10 \mu \mathrm{mol} / \mathrm{L}$ reverse primer, $10 \mu \mathrm{L} 2 \times$ SYBER Green master mix, and $5.5 \mu \mathrm{L} \mathrm{ddH}_{2} \mathrm{O}$
Table 1. The sequences of primers

\begin{tabular}{ll}
\hline Primer name & Sequence $\left(5^{\prime}-3^{\prime}\right)$ \\
\hline TNF- $\alpha$-forward & GAACTGGCAGAAGAGGCACT \\
TNF- - -reverse & GGTCTGGGCCATAGAACTGA \\
IL-6-forward & CTGATGCTGGTGACAACCAC \\
IL-6-reverse & CAGAATTGCCATTGCACAAC \\
ZO-1-forward & CCACCTCTGTCCAGCTCTTC \\
ZO-1-reverse & ACCGGAGTGATGGTTTTCTG \\
Beclin-1-forward & GGCCAATAAGATGGGTCTGA \\
Beclin-1-reverse & TGCACACAGTCCAGAAAAGC \\
atg5-forward & GATGGACAGCTGCACACACT \\
atg5-reverse & GCTGGGGGACAATGCTAATA \\
GAPDH-forward & AGTATGACTCCACTCACGGC \\
GAPDH-reverse & CACCAGTAGACTCCACGACA \\
\hline
\end{tabular}

TNF- $\alpha$, tumour necrosis factor- $\alpha$; IL-6, interleukin-6; ZO-1, zonula occludens-1; atg5, autophagy-related.

were prepared. qPCR was performed as follows: 2 min at $95^{\circ} \mathrm{C}$, 40 cycles of $15 \mathrm{~s}$ at $95^{\circ} \mathrm{C}, 25 \mathrm{~s}$ at $60^{\circ} \mathrm{C}$ and $60 \mathrm{~s}$ at $72^{\circ} \mathrm{C}$. The levels of tumour necrosis factor (TNF)- $\alpha$, IL- 6 , zonula occludens (ZO)-1, Beclin- 1 and atg 5 were determined by the $2^{-\Delta \Delta C T}$ method, and the internal reference for RNA was set as glyceraldehyde3-phosphate dehydrogenase (GAPDH). The primers used were shown in Table 1.

\section{Western Blot}

The total proteins of cells were extracted as aforementioned. After the proteins extraction, and Pierce ${ }^{\mathrm{TM}}$ BCA Protein Assay Kit (23250, Thermo Fisher, Waltham, MA, USA) was used to determine the concentration of the total proteins in each sample. Fifty micro gram total protein of each sample was loaded on the wells of sodium dodecyl sulfate-polyacrylamide gel, further separated at $100 \mathrm{~V}$ for $120 \mathrm{~min}$ and then transferred to PVDF membranes. Five per cent bovine serum albumin solution (B2064, Sigma-Aldrich, St. Louis, MO, USA) was used to block the membrane for $1 \mathrm{~h}$ and further probed with anti ZO-1 tight junction antibody (ab96587, Abcam, San Francisco, CA, USA, 187 kDa, 1:1,000), anti GAPDH antibody (ab8245, Abcam, San Francisco, CA, USA, 36 kDa, 1:1,000), anti-LC3B I antibody (ab48394, Abcam, San Francisco, CA, USA, $19 \mathrm{kDa}, 1: 1,000)$, anti-LC3B II antibody (ab48394, Abcam, San Francisco, CA, USA, $17 \mathrm{kDa}, 1: 1,000)$ at $4^{\circ} \mathrm{C}$ overnight. On the next day, the membranes were washed with tris-buffered saline tween (TBST, 28360, Thermo Fisher, Waltham, MA, USA) and further treated by the second antibody IgG H\&L (HRP; ab6721, Abcam, San Francisco, CA, USA, 1:2,000) at room temperature for $1 \mathrm{~h}$. The membranes were developed using Pierce ${ }^{\mathrm{TM}}$ ECL plus western blotting substrate (Thermo Fisher, Waltham, MA, USA) in ChemiDoc MP (Bio-Rad, Hercules, CA, USA). The value of gray in each band was analyzed in Image J software.

\section{Statistical Analysis}

All analysis was conducted using Prism Graphpad 7.0 software. The variables were demonstrated as mean \pm SD. Comparisons among multiple groups were performed using one-way analysis of variance. $p<0.05$ was considered statistically significant. 


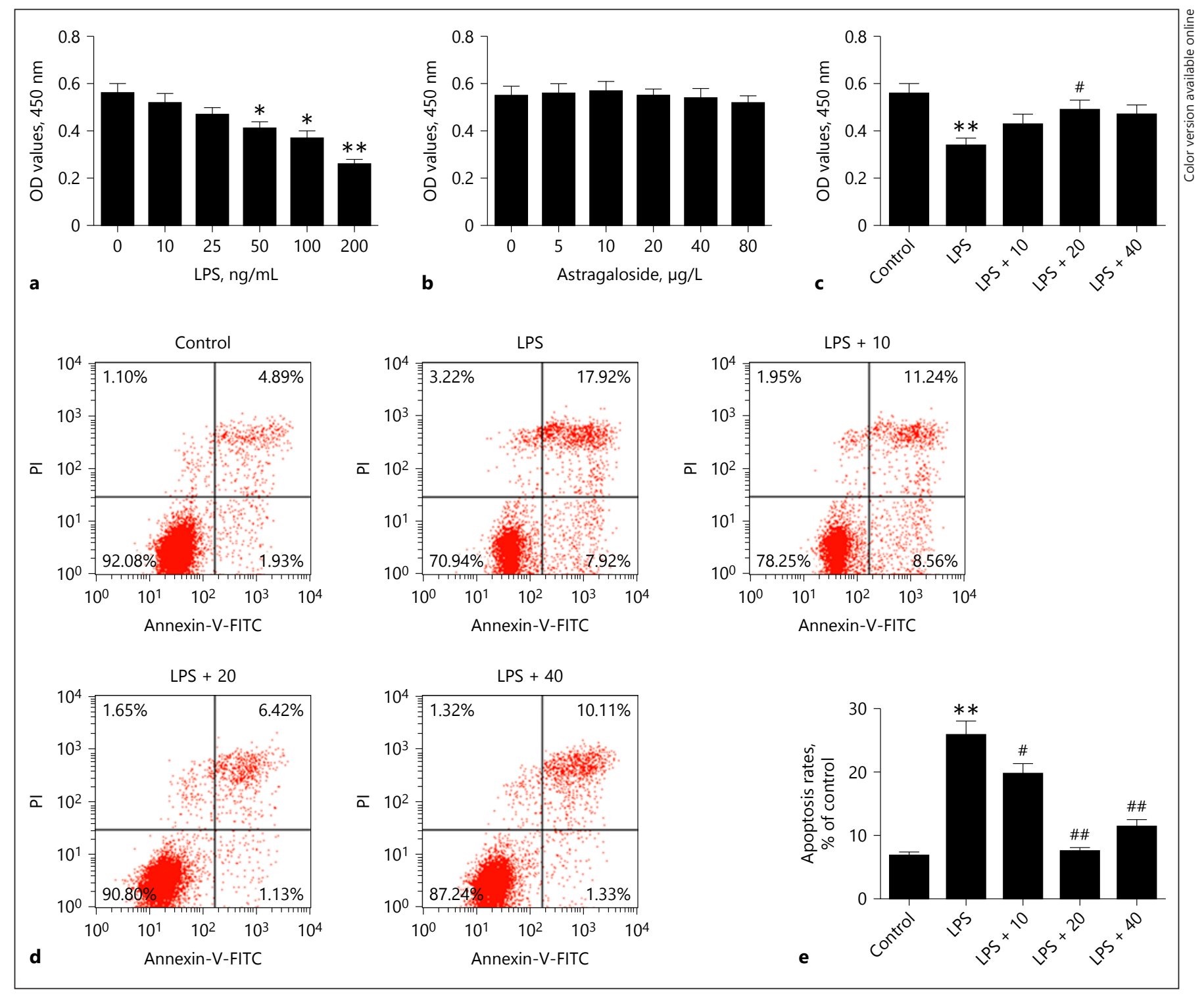

Fig. 1. The effect of LPS and astragaloside IV on cell viability and apoptosis of MLE-12 cells. a The ODat gradient concentration of LPS (ng/mL; b). The OD values at gradient concentration of astragaloside IV $(\mu \mathrm{g} / \mathrm{L} ; \mathbf{c})$. The OD values in control, LPS, LPS + 10, LPS +20 and LPS +40 groups. $\mathbf{d}$ The distribution of apoptotic cells

\section{Results}

\section{Astragaloside IV Enhanced the Cell Viability and}

Suppressed the Apoptosis Rate in LPS-Stimulated

\section{MLE-12 Cells}

To observe the effect of astragaloside IV on LPS-stimulated epithelial cells, we measured the changes of cell viability and apoptosis rate after the treating MLE-12 cells by LPS and astragaloside IV. Obviously, LPS at the con- on the diagram of flow cytometry. e The apoptosis rates (\% of control) in control, LPS, LPS + 10, LPS + 20 and LPS + 40 groups. Bars indicated mean \pm SD. ${ }^{* *} p<0.01$ and ${ }^{*} p<0.05 \mathrm{vs} .00 \mathrm{ng} / \mathrm{mL}$ LPS or $0 \mu \mathrm{g} / \mathrm{L}$ astragaloside IV or control group; ${ }^{\# \#} p<0.01$ and ${ }^{\#} p<$ 0.05 vs. LPS group. OD, optical density; LPS, lipopolysaccharides. ability of the cells (Fig. 1a, ${ }^{*} p<0.05$, ${ }^{* *} p<0.05$ ). However, the treatment of astragaloside IV alone could not affect the cell viability (Fig. 1b). Interestingly, we found that the cell viability in LPS +20 group was higher than in LPS group (Fig. 1c, ${ }^{* *} p<0.01,{ }^{*} p<0.05$ ). Moreover, the apoptosis rate in LPS group was higher than that in the control group but lower in LPS +10 , LPS +20 and LPS +40 groups compared with LPS group (Fig. 1e, ${ }^{* *} p<$ 


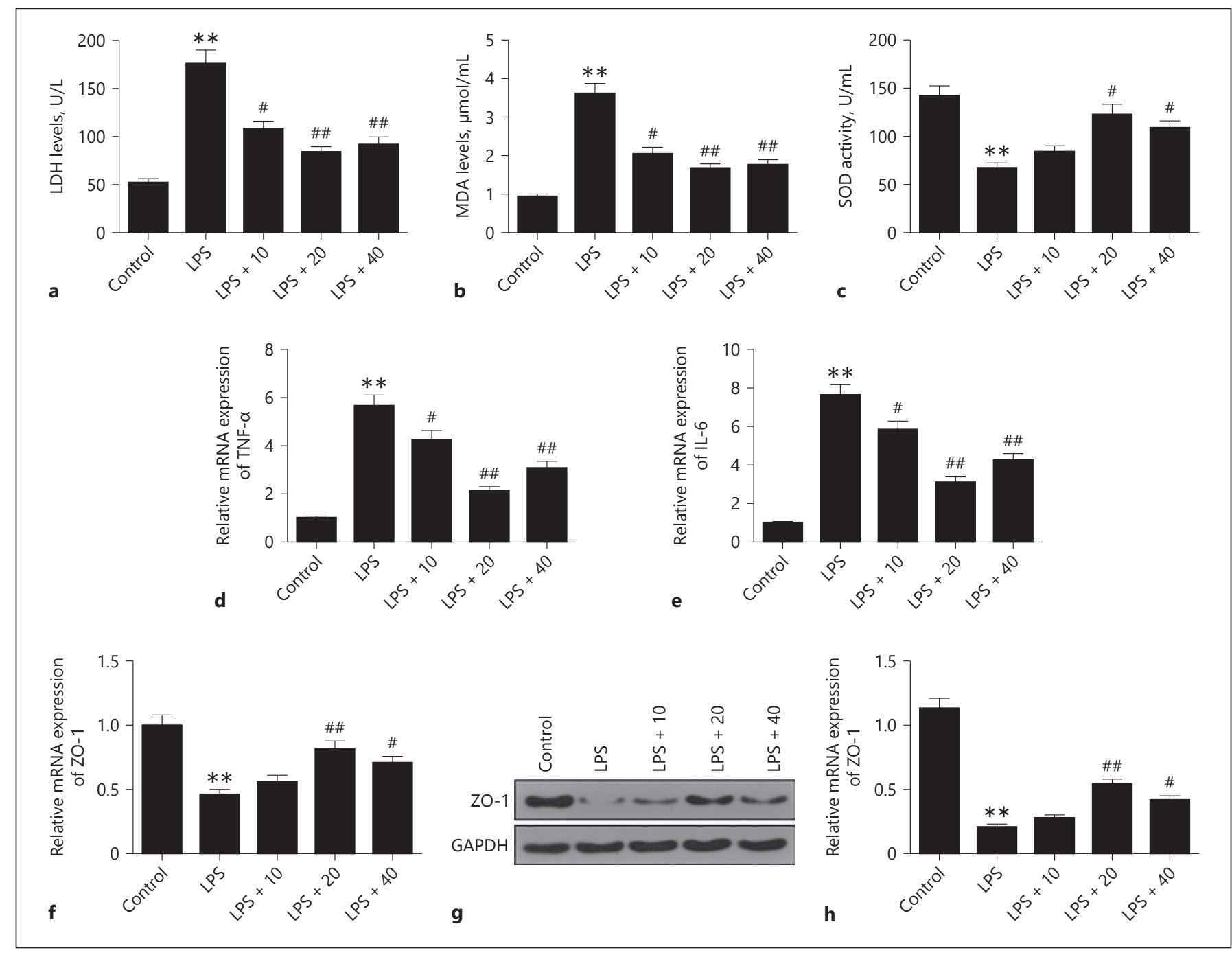

Fig. 2. The effect of astragaloside IV on LDH, MDA, SOD, TNF- $\alpha$, IL-6 and ZO-1 levels in control, LPS, LPS + 10, LPS + 20 and LPS + 40 groups of LPS-stimulated MLE- 12 cells. a The LDH levels (U/L) in each group. b The MDA levels $(\mu \mathrm{mol} / \mathrm{mL})$ in each group. $\mathbf{c}$ The SOD activity $(\mathrm{U} / \mathrm{mL})$ in each group. $\mathbf{d}$ The relative mRNA expression of TNF- $\alpha$ in each group. $\mathbf{e}$ The relative mRNA expression of IL- 6 in each group. $\mathbf{f}$ The relative mRNA expression of ZO-1 in

$\left.0.01,{ }^{\# \#} p<0.01,{ }^{\#} p<0.05\right)$. These data suggest that astragaloside IV contributes to the maintenance of pulmonary epithelial cells impaired by LPS.

\section{Astragaloside IV Suppressed Oxidative Stress and} Inflammatory Response and Enhanced Tight Junction in LPS-Stimulated MLE-12 Cells

For the observation of the effect of astragaloside IV in LPS-stimulated pulmonary epithelial cells, we measured LDH level, MDA level and SOD activity, the markers for each group. $\mathbf{g}$ The protein band of $\mathrm{ZO}-1$ in each group by Western Blot. $\mathbf{h}$ The relative protein level of $\mathrm{ZO}-1$ in each group. Bars indicated mean \pm SD. ${ }^{* *} p<0.01$ vs. control group; ${ }^{\# \#} p<0.01$ and ${ }^{\#} p<$ 0.05 vs. LPS group. LDH, lactate dehydrogenase; LPS, lipopolysaccharides; MDA, malondialdehyde; SOD, superoxide dismutase; TNF- $\alpha$, tumour necrosis factor- $\alpha$; IL-6, interleukin; ZO-1, zonula occludens. oxidative stress, and TNF- $\alpha$ and IL- 6 expressions, the inflammatory factors reflecting the condition of inflammatory response, as well as the expression of ZO-1, the tight junction factor indicating the formation of tight junction, in LPS-stimulated MLE-12 cells. Dramatically, we found that the LDH and MDA levels in the LPS group were higher than in control group, while they were lower in LPS + 10, LPS + 20 and LPS + 40 groups compared with the LPS group (Fig. 2a, b, ${ }^{* *} p<0.01$, \#\# $p<0.01$, ${ }^{\#} p<$ $0.05)$. Moreover, the SOD activity in the LPS group was
4

(n)

Liu/Zhao/Wang/Zhang/Ma 
lower than that in the control group but higher in LPS + 20 and LPS +40 groups than that in the LPS group (Fig. $2 c$, $\left.{ }^{* *} p<0.01,{ }^{*} p<0.05\right)$. Furthermore, the mRNA expression of TNF- $\alpha$ and IL- 6 in LPS group was higher than that in control groups but lower in LPS +10 , LPS +20 and LPS +40 groups than that in LPS groups (Fig. $2 \mathrm{~d}, \mathrm{e},{ }^{* *} p<$ $\left.0.01,{ }^{\# \#} p<0.01,{ }^{\#} p<0.05\right)$. In addition, the expression of ZO-1 in LPS group was lower than that in the control group but higher in LPS +20 and LPS +40 groups than that in the LPS group (Fig. $2 \mathrm{f}-\mathrm{h},{ }^{* *} p<0.01,{ }^{\# \#} p<0.01$, $\left.{ }^{\#} p<0.05\right)$. Taken together, the results indicate that astragaloside IV could decrease oxidative stress and inflammatory response and contribute to the formation of tight junction in pulmonary epithelial cells stimulated by LPS.

\section{Astragaloside IV Suppressed Autophagy in \\ LPS-Stimulated MLE-12 Cells}

To investigate the role of autophagy in the regulatory effect of astragaloside IV, we measured the expressions of LC3B I, LC3B II, Beclin-1 and atg 5 in LPS-stimulated MLE12 cells. Evidently, we found that LPS could reduce the expression of LC3B I from a concentration of $10 \mathrm{ng} / \mathrm{mL}$ while it could also raise the expression of LC3B II from a concentration of $25 \mathrm{ng} / \mathrm{mL}$ (Fig. 3a, b, ${ }^{* *} p<0.01,{ }^{*} p<0.05$ ). Similarly, LPS could elevate the expression of Beclin- 1 and atg 5 from a concentration of $50 \mathrm{ng} / \mathrm{mL}$ as well (Fig. $3 c$, ${ }^{* *} p<$ $0.01)$. Further, the expression of LC3B I was elevated and that of LC3B II was decreased in LPS + 10, LPS + 20 and LPS +40 groups compared to the LPS group (Fig. $3 \mathrm{~d}$, e, $\left.{ }^{* *} p<0.01,{ }^{\# \#} p<0.01\right)$. In addition, the expression of Beclin-1 in LPS + 20 was lower than that in the LPS group while the expression of atg 5 in LPS +20 and LPS +40 groups were less than that in the LPS group (Fig. 3f, ${ }^{* *} p<0.01$, \#\# $p<$ 0.01 ). It suggested that LPS could promote the autophagy in pulmonary epithelial cells, whereas astragaloside IV could suppress such an effect induced by LPS.

\section{Autophagy Regulated the Cell Viability and Tight}

\section{Junction in LPS-Stimulated MLE-12 Cells}

To investigate whether autophagy plays a critical role in the regulation of cell viability and tight junction formation, we measured the cell viability and the expressions of Beclin-1, atg5 and ZO-1 after pretreatment of LPS-stimulated MLE-12 cells by 3-MA and Rap. We found that the cell viability was higher in LPS + 3-MA but was lower in LPS + Rap groups than in the LPS group, and it was lower in the Rap group than that in the control group (Fig. 4a, $\left.{ }^{* *} p<0.01,{ }^{*} p<0.05,{ }^{*} p<0.05\right)$. Furthermore, the expressions of Beclin-1 and atg 5 were lower in LPS + 3-MA but were higher in LPS + Rap groups than in LPS groups, and they were lower in 3-MA but were higher in Rap groups than in control groups (Fig. $4 \mathrm{~b},{ }^{* *} p<0.01$, ${ }^{\# \#} p<$ $\left.0.01,{ }^{\#} p<0.05\right)$. In addition, the expression of ZO- 1 was higher in LPS + 3-MA but was lower in LPS + Rap groups than that in the LPS group; moreover, ZO-1 expression was decreased in the Rap group compared with that of the control group (Fig. 4c-e, ${ }^{* *} p<0.01,{ }^{\# \#} p<0.01$, ${ }^{\#} p<$ $0.05)$. Thus, the data suggest that the cell viability and tight junction in LPS-stimulated pulmonary epithelial cells could be modulated by autophagy.

\section{Astragaloside IV Regulated the Cell Viability and}

Tight Junction via Suppressing Autophagy in LPS-

Stimulated MLE-12 Cells

To explore whether autophagy is involved in the mechanism of astragaloside IV regulating the cell viability and tight junction, we measured the cell viability and the expressions of Beclin-1, atg5 and ZO-1 in LPS-stimulated MLE- 12 cells. Interestingly, the cell viability in LPS +20 group was higher than that in the LPS group; however, it was decreased in the LPS + $20+$ Rap group compared with that of the LPS +20 group (Fig. $5 \mathrm{a},{ }^{* *} p<0.01,{ }^{\#} p<$ $\left.0.05,{ }^{\&} p<0.05\right)$. Moreover, the expressions of Beclin-1 and atg 5 in LPS +20 group were lower than in those in the LPS group, and there was no significant difference among LPS + 20, LPS + $20+3$-MA and LPS + $20+$ Rap groups (Fig. $5 b,{ }^{* *} p<0.01,{ }^{\#} p<0.05$ ). In addition, the expression of ZO-1 in LPS + 20 group was higher than that in the LPS group but was lowered in the LPS + $20+$ Rap group compared with that of the LPS +20 group (Fig. $\left.5 c-e,{ }^{* *} p<0.01,{ }^{\& \&} p<0.01,{ }^{*} p<0.05\right)$. Therefore, it suggests that by suppressing the autophagy, astragaloside IV exerts its regulatory function in LPS-stimulated pulmonary epithelial cells, thereby maintaining the cell viability and tight junction to some extent.

\section{Discussion}

In the present study, we explored the effects of astragaloside IV on cell viability, apoptosis, oxidative stress, inflammatory response and tight junction in LPS-stimulated MLE- 12 cells, and found that autophagy acted as a regulator in the effect of astragaloside IV, indicating that astragaloside IV has a potential pharmaceutical mechanism in the interference of ARDS in the cell model.

Here, we used LPS to stimulate MLE-12 cells to construct an ARDS model in vitro as previously described [4, 20]. LPS can activate nuclear factor-kappa B pathway through a series of signal transduction, and lead to the re- 

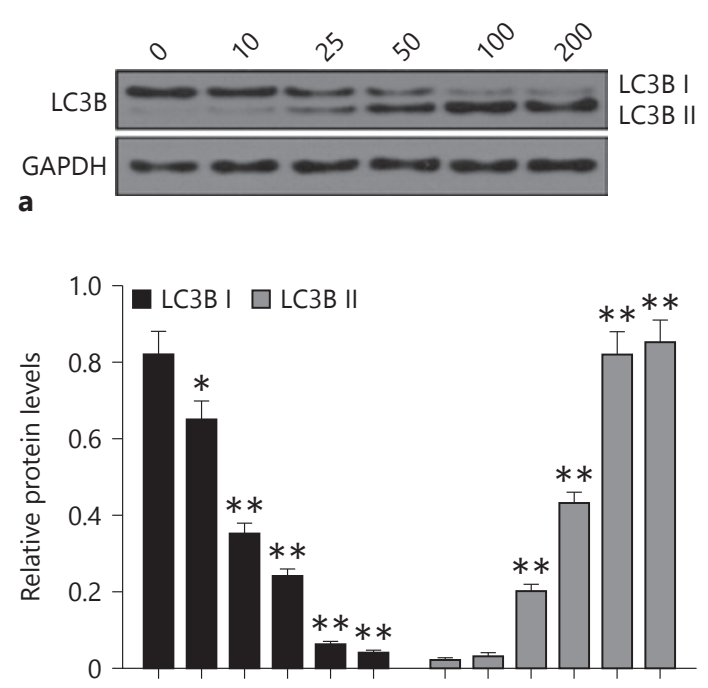

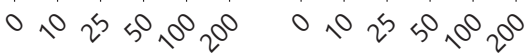

b

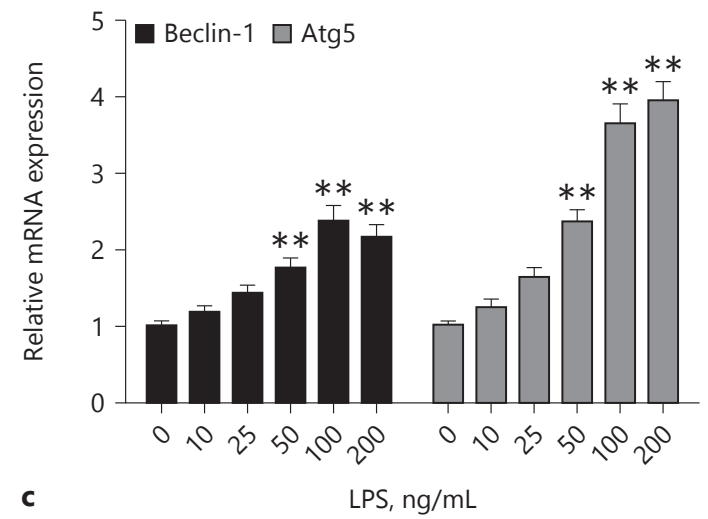

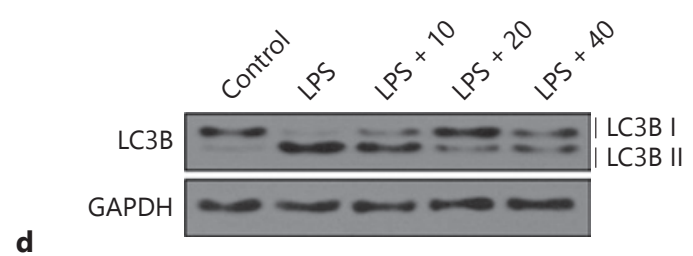
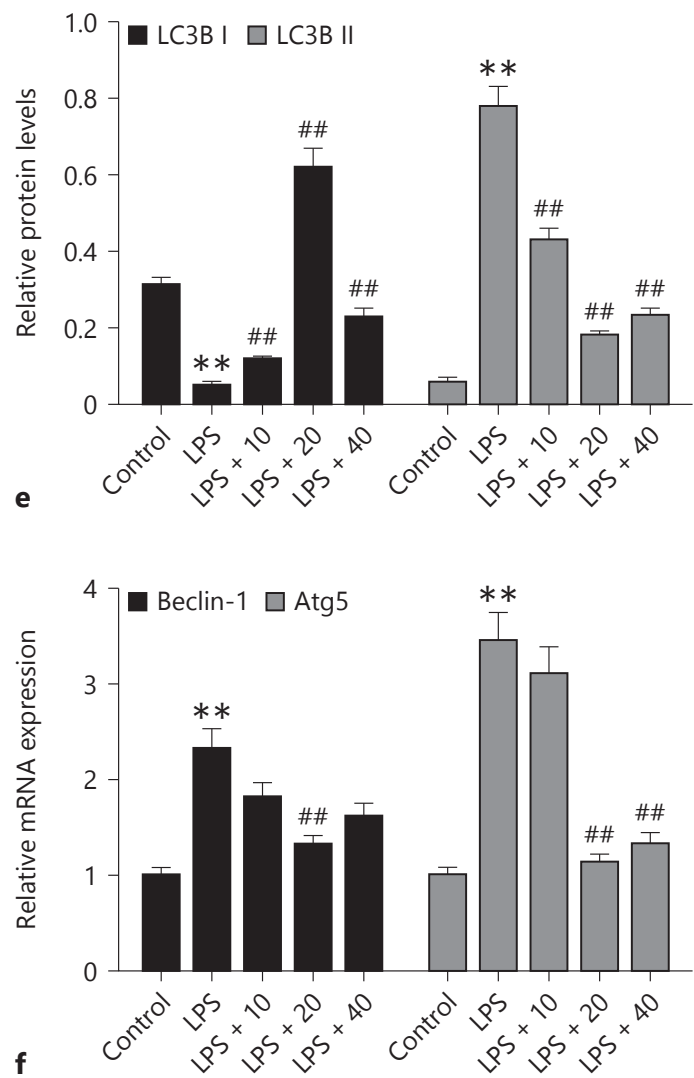

Fig. 3. The effect of LPS and astragaloside IV on the expressions of microtubule-associated proteins 1A/1B LC3B I, LC3B II, Beclin-1 and atg5. a The protein band of $\mathrm{LC} 3 \mathrm{~B}$ at gradient concentration of LPS (ng/mL) by Western Blot. $\mathbf{b}$ The relative protein level of LC3B I and II at gradient concentration of LPS $(\mathrm{ng} / \mathrm{mL} ; \mathbf{c})$. The relative mRNA expression of Beclin-1 and atg 5 at gradient concentration of LPS (ng/mL; d). The protein band of LC3B in control, LPS, LPS +

lease of a large number of inflammatory mediators, which is one of the important factors causing ARDS [21,22]. We found that LPS could lower the cell viability and increase the apoptosis rate in MLE-12 cell line, and such effects could be reversed partially by astragaloside IV. However, treating the MLE-12 cells by astragaloside IV alone had no effect, suggesting that astragaloside IV could only be functional in LPS-stimulated cells. Qi et al. [4] showed that the cell viability was reduced, but the apoptosis rate was in-
10, LPS + 20 and LPS + 40 groups by Western blot. e The relative protein level of LC3B I and II in control, LPS, LPS + 10, LPS + 20 and LPS +40 groups. $\mathbf{f}$ The relative mRNA expression of Beclin-1 and atg 5 in control, LPS, LPS + 10, LPS + 20 and LPS + 40 groups. Bars indicated mean \pm SD. ${ }^{* *} p<0.01$ and ${ }^{*} p<0.05 \mathrm{vs.0} 0 \mathrm{ng} / \mathrm{mL}$ LPS or control group; ${ }^{\# \#} p<0.01$ vs. LPS group. LC3B, light chain 3B; LPS, lipopolysaccharides; atg 5 , autophagy-related 5.

creased in the LPS-induced ARDS cell model. Wang et al. [23] revealed that cell viability of LPS-induced H9C2 cardiomyocytes was increased by the treatment of astragaloside IV. Moreover, Liu et al. [24] reported that a high dose of astragaloside IV greatly protected against acute liver injury in D-GalN/LPS-induced mice and suppressed apoptosis of hepatocytes. Therefore, we speculated that astragaloside IV was able to prevent the pulmonary epithelial cells from the impairment caused by LPS. 


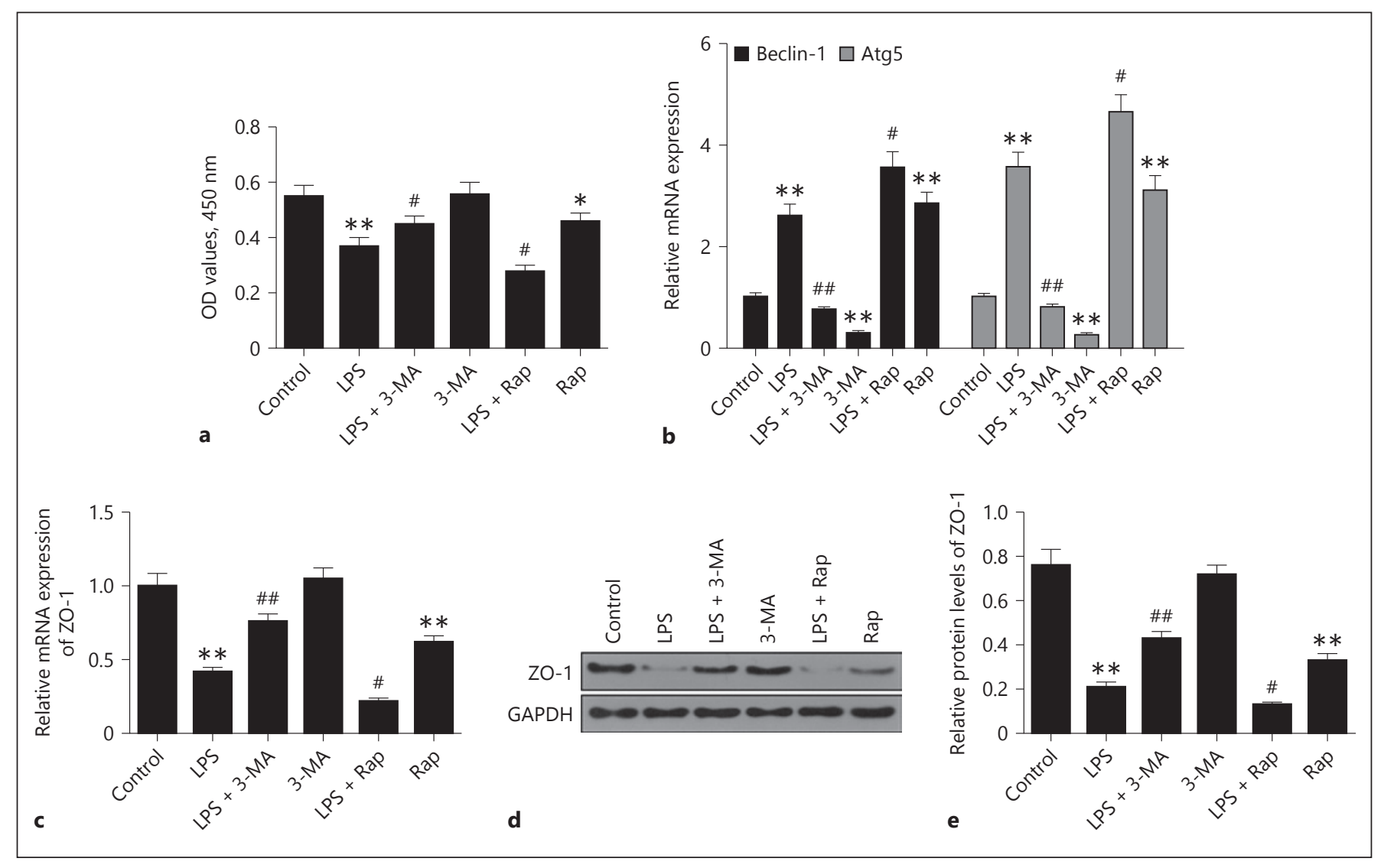

Fig. 4. The alterations of cell viability and the expressions of Beclin-1, atg 5 and ZO-1 after using 3-MA and Rap in LPS-stimulated MLE- 12 cells. a The OD values in control, LPS, LPS + 3-MA, 3-MA, LPS + Rap and Rap groups. $\mathbf{b}$ The relative mRNA expressions of Beclin-1 and atg5 in control, LPS, LPS + 3-MA, 3-MA, LPS + Rap and Rap groups. $c$ The relative mRNA expression of ZO- 1 in control, LPS, LPS + 3-MA, 3-MA, LPS + Rap and Rap groups. d The protein bands of ZO-1 in control, LPS, LPS + 3-MA, 3-MA, LPS + Rap and Rap groups by Western blot. e The relative protein level of ZO-1 in control, LPS, LPS + 3-MA, 3-MA, LPS + Rap and Rap groups. Bars indicated mean \pm SD. ${ }^{* *} p<0.01$ and ${ }^{*} p<0.05$ vs. control group; ${ }^{\# \#} p<0.01$ and ${ }^{\#} p<0.05$ vs. LPS group. OD, optical density; LPS, lipopolysaccharides; MA, methyladenine; Rap, Rapamycin; atg5, autophagy-related 5; ZO-1, zonula occludens.
Chen et al. [3] showed that oxidative stress and inflammation were triggered in LPS-induced pulmonary endothelial cell model. LDH, MDA and SOD are widely studied biomarkers that represent the status of oxidative stress $[25,26]$. In the early stage of lung injury, TNF- $\alpha$ and IL-6 could not only participate in the inflammatory reaction and damage the vascular endothelium, but also cooperate with thrombin to promote coagulation to induce ARDS [27]. Previous studies have shown that during the development of ARDS, tight junction protein expression and distribution will change, which enhances the selective permeation of fluids and proteins via the paracellular pathway, thereby leading to pulmonary oedema $[8,28]$. $\mathrm{ZO}-1$ is associated to the cytoskeleton through transmembrane protein and participates in the formation of tight junction complexes $[29,30]$. Here, we discovered that the levels of LDH and MDA and the expressions of TNF- $\alpha$ and IL- 6 were up-regulated by LPS but were down-regulated by astragaloside IV. Meanwhile, SOD activity and ZO-1 expression were decreased by LPS, however, they were up-regulated by astragaloside IV. In the present study, TNF- $\alpha$ and IL-6 are mainly derived from pulmonary epithelial cells. Pooladanda et al. [31] reported that TNF- $\alpha$ played a key role in LPS-induced pulmonary epithelial cell model, in which oxidative stress and inflammation were triggered. Xie et al. [7] reported that ZO-1 protein expression was reduced in LPS-treated mice. Zhang et al. [32] also found that LPS treatment decreased the ZO-1 expression in human pulmonary microvascular endothelial cells, which was related to the high permeability induced by LPS. However, further studies are required to determine the specific mechanism by which LPS regulates 


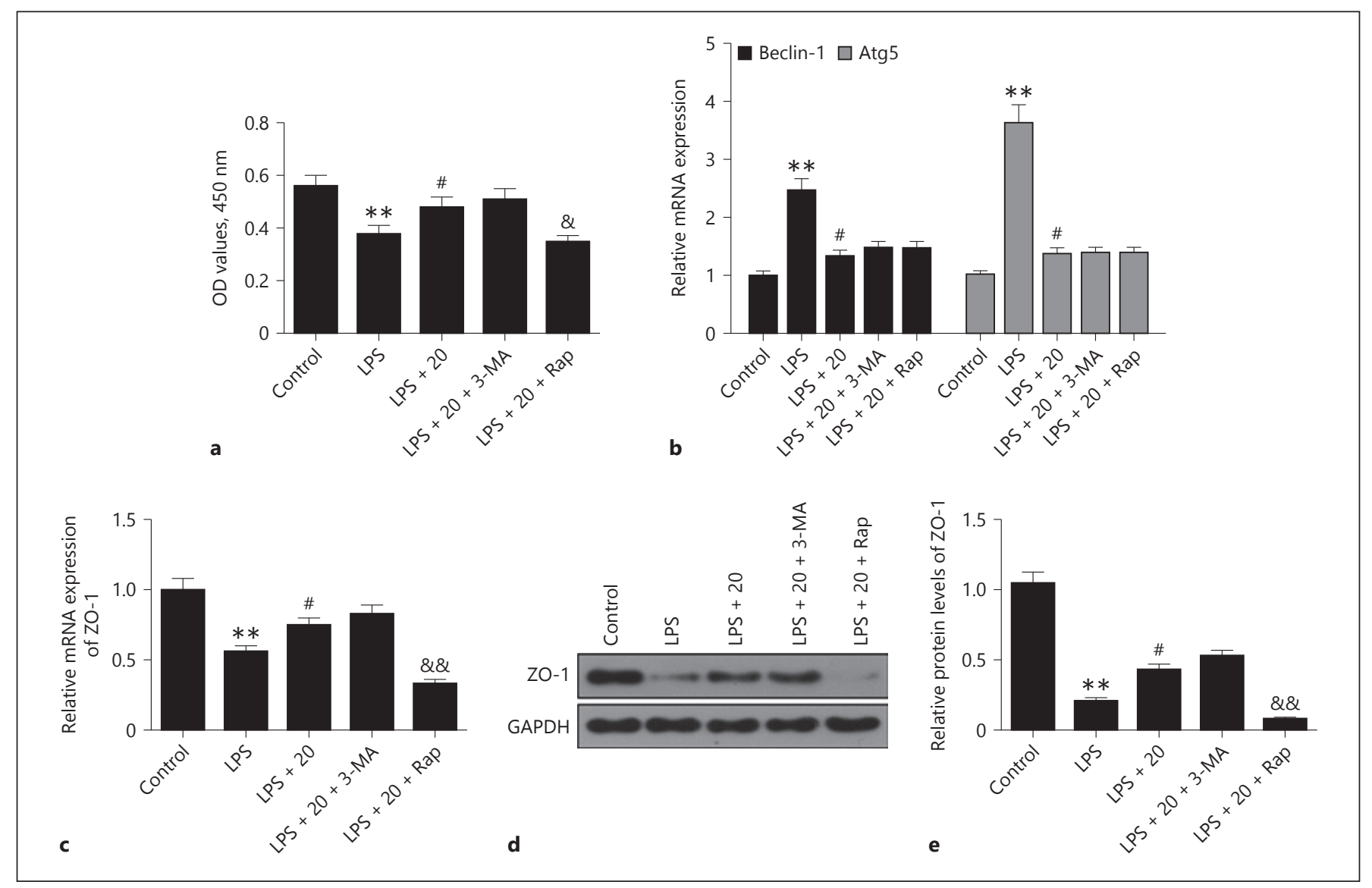

Fig. 5. The effect of astragaloside IV on cell viability and the expressions of Beclin-1, atg 5 and ZO-1 in control, LPS, LPS +20 , LPS + $20+3$-MA and LPS + $20+$ Rap groups of LPS-stimulated MLE-12 cells in the presence of 3-MA or Rap. a The OD values in each group. $\mathbf{b}$ The relative mRNA expression of Beclin- 1 and atg 5 in each group. $\mathbf{c}$ The relative mRNA expression of $\mathrm{ZO}-1$ in each

ZO-1 expression. Yu et al. [33] demonstrated that Astragaloside IV inhibited oxidative stress and inflammation in rats with pulmonary fibrosis. Li et al. [34] revealed that astragaloside IV prevented the integrity of bloodbrain-barrier, which was composed of endothelial cells in cerebral microvessels and connected by tight junctions, from LPS-induced disruption. Qu et al. [35] suggest that astragaloside IV contributes to the maintenance of tight junction, which is reflected by the elevation of ZO-1 expression in the blood-brain barrier protection in the ischemia/reperfusion rats. Thus, we speculate that astragaloside IV contributes to the reduction of oxidative stress, inflammatory response and tight junction in LPS-stimulated pulmonary epithelial ARDS cell model.

Beclin-1 is homologous to the yeast autophagy gene atg6 and is a key factor regulating the formation of au- group. $\mathbf{d}$ The protein bands of ZO- 1 in each group. $\mathbf{e}$ The relative protein level of ZO- 1 in each group. Bars indicated mean \pm SD. ${ }^{* *} p<0.01$ vs. control group; ${ }^{\#} p<0.05$ vs. LPS group; $\& \& p<0.01$ and ${ }^{\&} p<0.05$ vs. LPS +20 group. OD, optical density; LPS, lipopolysaccharides; MA, methyladenine; Rap, Rapamycin; atg5, autophagy-related 5; ZO-1, zonula occludens. tophagosomes in mammals [36]. LC3 (including LC3B I and II) is also a key gene involved in autophagy, and as LC3 content is closely related to autophagy activity, it is seen as a marker of autophagy $[37,38]$. Atg5 is a key factor in the formation of autosomal bodies in the ubiquitinlike junction system atgl2-atg5-atgl6 and is proved to be essential for autophagosome formation $[39,40]$. Here, we found that LC3B I expression was decreased, while LC3B II, Beclin-1 and atg5 expressions were elevated by LPS. However, these trends could be partially reversed by astragaloside IV, indicating that astragaloside IV partly offset the increased autophagy by LPS in pulmonary epithelial cells. In the current study, LPS may induce autophagy by promoting oxidative stress and inflammatory response of pulmonary epithelial cells. At present, mitochondrial damage, ROS generation and excessive inflammatory cy- 
tokines are believed to be involved in the process of LPSinduced autophagy $[32,41]$. Future studies should further clarify the exact mechanism of LPS-induced autophagy in lung epithelial cells. Dong et al. [42] revealed that astragaloside IV reduced excessive autophagy after heat induction in vitro and in vivo. Xu et al. [43] also demonstrated that astragaloside IV reversed the autophagy in mice induced by the intestinal microbiota of acute ischaemic stroke. Previous research proved that oxidative stress can induce autophagy activation through a variety of mechanisms [44]. Moreover, a close relationship between autophagy and inflammation was observed, and TNF- $\alpha$ and interferon $-\gamma$ were considered to be autophagy inducers [12]. Thus, it could be speculated that astragaloside IV could suppress the initiation of autophagy by LPS directly or indirectly through suppressing the oxidative stress and inflammatory response in LPS-induced pulmonary endothelial ARDS cell model.

To further determine the modulation of autophagy on cell viability and tight junction in LPS-induced ARDS cell model, we inhibited and induced autophagy by 3 -MA and Rap. Here, we found that inhibiting autophagy increased the cell viability, while inducing autophagy decreased ZO-1 expression. Bi et al. [45] found that lycopene inhibits autophagy in human cSCC cells by activating ERK, JNK and MTORC1 and up-regulating the ZO-1 expression. A study reported that inhibition of autophagy further aggravates the decreased expression of ZO-1 in human pulmonary microvascular endothelial cells induced by LPS [32]. Here, we found that the inhibition of autophagy partially reversed the down-regulation of ZO-1 in lung epithelial cells induced by LPS; however, the possible mechanism of inhibiting ZO-1 expression by the inhibition of autophagy remains to be further explored. Lo et al. [46] showed that autophagy had cytotoxic effect, which affects the cell viability in osteosarcoma cells. Nighot et al. [47] revealed that intestinal epithelial tight junction was enhanced by autophagy. Therefore, it could be reasoned that autophagy could regulate the cell viability and tight junction of pulmonary epithelial cells.

Based on the previous outcomes, we hypothesized that autophagy possibly act as a mediator in the effect of astragaloside IV and further proved the hypothesis using 3-MA and Rap in the cells treated by LPS and astragaloside IV. Here, we found that Rap partially reversed the effect of Astragaloside IV on LPS-induced cell activity and ZO-1, whereas 3-MA had no effect on Astragaloside IV. Moreover, Astragaloside inhibition of Beclin-1 and atg5 expression was not affected by 3-MA or Rap, suggesting that autophagy could be suppressed by astragalo- side IV and further increases the cell viability and tight junction of pulmonary endothelial cells impaired by LPS. Wu et al. [48] revealed that inhibition of autophagy reduced the severity of LPS-induced acute kidney injury.

This study has some limitations, for example, the effect of inhibition of autophagy on production of TNF- $\alpha$ and IL-6 remains to be further explored. In addition, previous studies confirmed that the toxic effects of LPS were dependent on the expressions of LPS receptors; however, the expressions of LPS receptors and the effect of LPS \pm astragaloside IV were not clarified in the current study. Furthermore, astragaloside IV reducing lung epithelial cell injury by inhibiting autophagy needs to be further discussed in vivo using experimental animal models and different cell lines.

In conclusion, astragaloside IV might suppress the initiation of autophagy directly or indirectly through suppressing the oxidative stress and inflammatory response, and further increases the cell viability and tight junction and reduces apoptosis in LPS-stimulated pulmonary endothelial ARDS cell model, thereby exerting its therapeutic function in ARDS.

\section{Acknowledgements}

This work was supported by National Natural Science Foundation of China (Grant number: 81102553); the Key International Cooperation Project of Shanxi Province (Grant number: 201603D421018); the Project of Health and Family Planning Commission of the Shanxi Province (Grant number: 201601102).

\section{Statement of Ethics}

No humans were involved in this research.

\section{Disclosure Statement}

The authors declare that they have no conflicts of interest to disclose.

\section{Author Contributions}

B.L., H. Zhao, and Y.W.: substantial contributions to conception and design. H. Zhang, Y.M. and B.L.: data acquisition, data analysis and interpretation. B.L., H. Zhang, and H. Zhao.: drafting the article or critically revising it for important intellectual content. All authors: final approval of the version to be published. Y.M. and Y.W.: agreement to be accountable for all aspects of the work in ensuring that questions related to the accuracy or integrity of the work are appropriately investigated and resolved. 


\section{References}

1 Ware LB, Matthay MA. The acute respiratory distress syndrome. N Engl J Med. 2000 May; 342(18):1334-49.

2 Jiang Y, Zeng Y, Huang X, Qin Y, Luo W, Xiang S, et al. Nur77 attenuates endothelin-1 expression via downregulation of NF- $\mathrm{KB}$ and p38 MAPK in A549 cells and in an ARDS rat model. Am J Physiol Lung Cell Mol Physiol. 2016 Dec;311(6):L1023-35.

3 Chen L, Li W, Qi D, Wang D. Lycium barbarum polysaccharide protects against LPSinduced ARDS by inhibiting apoptosis, oxidative stress, and inflammation in pulmonary endothelial cells. Free Radic Res. 2018 Apr; 52(4):480-90.

4 Qi D, Wang D, Zhang C, Tang X, He J, Zhao $\mathrm{Y}$, et al. Vaspin protects against LPS-induced ARDS by inhibiting inflammation, apoptosis and reactive oxygen species generation in pulmonary endothelial cells via the Akt/GSK-3 $\beta$ pathway. Int J Mol Med. 2017 Dec;40(6): 1803-17.

5 Baetz D, Shaw J, Kirshenbaum LA. Nuclear factor-kappaB decoys suppress endotoxin-induced lung injury. Mol Pharmacol. 2005 Apr; 67(4):977-9.

6 Yu J, Wang Y, Li Z, Dong S, Wang D, Gong L, et al. Effect of Heme Oxygenase-1 on Mitofusin-1 protein in LPS-induced ALI/ARDS in rats. Sci Rep. 2016 Nov;6(1):36530.

7 Xie W, Wang H, Wang L, Yao C, Yuan R, Wu $\mathrm{Q}$. Resolvin D1 reduces deterioration of tight junction proteins by upregulating $\mathrm{HO}-1$ in LPS-induced mice. Lab Invest. 2013 Sep; 93(9):991-1000.

8 Wang ZH, Ren WY, Zhu L, Hu LJ. Plasminogen activator inhibitor-1 regulates LPS induced inflammation in rat macrophages through autophagy activation. ScientificWorldJournal. 2014;2014:189168.

9 Sarma JV, Ward PA. Oxidants and redox signaling in acute lung injury. Compr Physiol. 2011 Jul;1(3):1365-81.

10 Lin X, Barravecchia M, Kothari P, Young JL, Dean DA. $\beta 1-\mathrm{Na}(+), \mathrm{K}(+)$-ATPase gene therapy upregulates tight junctions to rescue lipopolysaccharide-induced acute lung injury. Gene Ther. 2016 Jun;23(6):489-99.

11 Wittekindt $\mathrm{OH}$. Tight junctions in pulmonary epithelia during lung inflammation. Pflugers Arch. 2017 Jan;469(1):135-47.

12 Jones SA, Mills KH, Harris J. Autophagy and inflammatory diseases. Immunol Cell Biol. 2013 Mar;91(3):250-8.

13 Sun Y, Li C, Shu Y, Ju X, Zou Z, Wang H, et al. Inhibition of autophagy ameliorates acute lung injury caused by avian influenza A H5N1 infection. Sci Signal. 2012 Feb;5(212):ra16.

14 Rojo de la Vega M, Dodson M, Gross C, Mansour HM, Lantz RC, Chapman E, et al. Role of Nrf2 and Autophagy in Acute Lung Injury. Curr Pharmacol Rep. 2016 Apr;2(2): 91-101.

15 Li X, Qu L, Dong Y, Han L, Liu E, Fang S, et al. A review of recent research progress on the astragalus genus. Molecules. 2014 Nov; 19(11):18850-80.

16 Zhang ZG, Wu L, Wang JL, Yang JD, Zhang J, Zhang J, et al. Astragaloside IV prevents MPP囚-induced SH-SY5Y cell death via the inhibition of Bax-mediated pathways and ROS production. Mol Cell Biochem. 2012 May; 364(1-2):209-16.

17 Chen T, Wang R, Jiang W, Wang H, Xu A, Lu $\mathrm{G}$, et al. Protective Effect of Astragaloside IV Against Paraquat-Induced Lung Injury in Mice by Suppressing Rho Signaling. Inflammation. 2016 Feb;39(1):483-92.

18 Wikenheiser KA, Vorbroker DK, Rice WR, Clark JC, Bachurski CJ, Oie HK, et al. Production of immortalized distal respiratory epithelial cell lines from surfactant protein $\mathrm{C} /$ simian virus 40 large tumor antigen transgenic mice. Proc Natl Acad Sci USA. 1993 Dec;90(23): 11029-33.

19 Qi D, He J, Wang D, Deng W, Zhao Y, Ye Y, et al. $17 \beta$-estradiol suppresses lipopolysaccharide-induced acute lung injury through PI3K/Akt/SGK1 mediated up-regulation of epithelial sodium channel $(\mathrm{ENaC})$ in vivo and in vitro. Respir Res. 2014 Dec;15(1):159.

20 Qi D, Tang X, He J, Wang D, Zhao Y, Deng $\mathrm{W}$, et al. Omentin protects against LPS-induced ARDS through suppressing pulmonary inflammation and promoting endothelial barrier via an Akt/eNOS-dependent mechanism. Cell Death Dis. 2016 Sep;7(9):e2360.

21 Martin TR. Direct lung injury by bacteria: clarifying the tools of the trade. Crit Care Med. 2004 Nov;32(11):2360-1.

22 Liu N, Liu X, Li X, Duan K, Deng Y, Yu X, et al. DOK3 Degradation is Required for the Development of LPS-induced ARDS in Mice. Curr Gene Ther. 2016;16(4):256-62.

23 Wang SG, Xu Y, Xie H, Wang W, Chen XH. Astragaloside IV prevents lipopolysaccharide-induced injury in H9C2 cardiomyocytes. Chin J Nat Med. 2015 Feb;13(2):127-32.

24 Liu L, Li SJ, Zhou Y. [Protective effect of astragaloside IV against acute liver failure in experimental mice]. Zhonghua Gan Zang Bing Za Zhi. 2016 Oct 20;24(10):772-7.

25 Goncalves JM, Casart YC, Camejo MI. Nitric oxide and oxidative stress in placental explant cultures. Syst Biol Reprod Med. 2016;62(1): 11-6.

26 Samarghandian S, Azimi-Nezhad M, Farkhondeh T, Samini F. Anti-oxidative effects of curcumin on immobilization-induced oxidative stress in rat brain, liver and kidney. Biomed Pharmacother. 2017 Mar;87:223-9.

27 MacLaren R, Stringer KA. Emerging role of anticoagulants and fibrinolytics in the treatment of acute respiratory distress syndrome. Pharmacotherapy. 2007 Jun;27(6):860-73.

28 Uchida T. Acute lung injury and alveolar epithelial function. J Anesth. 2011 Feb;25(1): 152-4.

29 Chiba H, Osanai M, Murata M, Kojima T, Sawada N. Transmembrane proteins of tight junctions. Biochim Biophys Acta. 2008 Mar; 1778(3):588-600.

30 González-Mariscal L, Domínguez-Calderón A, Raya-Sandino A, Ortega-Olvera JM, Vargas-Sierra O, Martínez-Revollar G. Tight junctions and the regulation of gene expression. Semin Cell Dev Biol. 2014 Dec;36:21323.

31 Pooladanda V, Thatikonda S, Bale S, Pattnaik B, Sigalapalli DK, Bathini NB, et al. Nimbolide protects against endotoxin-induced acute respiratory distress syndrome by inhibiting TNF- $\alpha$ mediated NF- $\kappa B$ and HDAC- 3 nuclear translocation. Cell Death Dis. 2019 Jan; 10(2):81.

32 Zhang D, Zhou J, Ye LC, Li J, Wu Z, Li Y, et al. Autophagy maintains the integrity of endothelial barrier in LPS-induced lung injury. J Cell Physiol. 2018 Jan;233(1):688-98.

33 Yu WN, Sun LF, Yang H. Inhibitory Effects of Astragaloside IV on Bleomycin-Induced Pulmonary Fibrosis in Rats Via Attenuation of Oxidative Stress and Inflammation. Inflammation. 2016 Oct;39(5):1835-41.

34 Li H, Wang P, Huang F, Jin J, Wu H, Zhang $\mathrm{B}$, et al. Astragaloside IV protects blood-brain barrier integrity from LPS-induced disruption via activating Nrf2 antioxidant signaling pathway in mice. Toxicol Appl Pharmacol. 2018 Feb;340:58-66.

35 Qu YZ, Li M, Zhao YL, Zhao ZW, Wei XY, Liu JP, et al. Astragaloside IV attenuates cerebral ischemia-reperfusion-induced increase in permeability of the blood-brain barrier in rats. Eur J Pharmacol. 2009 Mar;606(1-3): 137-41.

36 Sun Q, Fan W, Zhong Q. Regulation of Beclin 1 in autophagy. Autophagy. 2009 Jul;5(5): 713-6.

37 Shpilka T, Weidberg H, Pietrokovski S, Elazar Z. Atg8: an autophagy-related ubiquitin-like protein family. Genome Biol. 2011 Jul;12(7): 226.

38 Mizushima N, Yoshimori T, Levine B. Methods in mammalian autophagy research. Cell. 2010 Feb;140(3):313-26.

39 Walczak M, Martens S. Dissecting the role of the Atg12-Atg5-Atg16 complex during autophagosome formation. Autophagy. 2013 Mar;9(3):424-5.

40 Li X, Li C, Zhu L. Correlation of autophagyassociated gene Atg5 with tumorigenesis of prostate cancer. Zhonghua Nan Ke Xue. 2015 Jan;21(1):31-4.

41 Yuan H, Perry CN, Huang C, Iwai-Kanai E, Carreira RS, Glembotski CC, et al. LPS-induced autophagy is mediated by oxidative signaling in cardiomyocytes and is associated with cytoprotection. Am J Physiol Heart Circ Physiol. 2009 Feb;296(2):H470-9.

42 Dong Z, Zhou J, Zhang Y, Chen Y, Yang Z, Huang G, et al. Astragaloside-IV Alleviates Heat-Induced Inflammation by Inhibiting Endoplasmic Reticulum Stress and Autophagy. Cell Physiol Biochem. 2017;42(2):824-37. 
43 Xu N, Kan P, Yao X, Yang P, Wang J, Xiang $\mathrm{L}$, et al. Astragaloside IV reversed the autophagy and oxidative stress induced by the intestinal microbiota of AIS in mice. J Microbiol. 2018 Nov;56(11):838-46.

44 Filomeni G, De Zio D, Cecconi F. Oxidative stress and autophagy: the clash between damage and metabolic needs. Cell Death Differ. 2015 Mar;22(3):377-88.
45 Bi S, Li L, Gu H, Li M, Xu S, Bu W, et al. Lycopene upregulates $\mathrm{ZO}-1$ and downregulates claudin-1 through autophagy inhibition in the human cutaneous squamous cell carcinoma cell line COLO-16. J Cancer. 2019 Jan; 10(2):510-21.

46 Lo YC, Lin YC, Huang YF, Hsieh CP, Wu CC, Chang IL, et al. Carnosol-Induced ROS Inhibits Cell Viability of Human Osteosarcoma by Apoptosis and Autophagy. Am J Chin Med. 2017;45(8):1761-72.
47 Nighot PK, Hu CA, Ma TY. Autophagy enhances intestinal epithelial tight junction barrier function by targeting claudin-2 protein degradation. J Biol Chem. 2015 Mar;290(11): 7234-46.

48 Wu Y, Zhang Y, Wang L, Diao Z, Liu W. The Role of Autophagy in Kidney Inflammatory Injury via the NF- $\mathrm{kB}$ Route Induced by LPS Int J Med Sci. 2015 Aug;12(8):655-67. 